\title{
ON UNICOHERENT CONTINUA
}

\author{
BY \\ HARLAN C. MILLER
}

The first section of this paper deals mainly with hereditarily unicoherent continua which are atriodic. The term "continuum" will imply compactness. A continuum $M$ is said to be unicoherent if the set of all points common to any two continua whose sum is $M$ is connected. It is said to be atriodic if it does not contain three continua such that the common part of each pair is the common part of all three and is a proper subcontinuum of each. A property of a continuum which is possessed by all its subcontinua is said to be hereditary. The second section is chiefly concerned with conditions implying hereditary unicoherence. Among other things it is proved that in order that a hereditarily decomposable continuum be hereditarily unicoherent it is necessary and sufficient that it contain no continuum $N$ which can be decomposed into an upper semi-continuous collection of mutually exclusive continua with respect to the elements of which $N$ is a simple closed curve. In establishing this theorem it is proved that a hereditarily decomposable irreducible continuum $M$ can be decomposed into an upper semi-continuous collection with respect to the elements of which $M$ is an arc. The third section is devoted to properties of a continuum every subcontinuum of which is unicoherent and decomposable which are analogous to certain properties of an acyclic continuous curve.

The basic space is a Moore space and, since all the theorems deal with internal properties of continua, may therefore be described as metric. The reader is referred to R. L. Moore's Foundations of point set theory ${ }^{1}$ ) for definitions of terms not defined here.

\section{Atriodic hereditarily unicoherent continua.}

THEOREM 1.1. In order that the continuum $M$ be hereditarily unicoherent, it is necessary and sufficient that if $A$ and $B$ are two points of $M$, there is only one irreducible subcontinuum( $\left.{ }^{2}\right) A B$ of $M$.

Presented to the society, February 25,1939, under the title Some methods of characterizing certain atriodic continua, January 1, 1941, under the title Concerning certain types of end points, and April 12, 1941, under the title Concerning compact unicoherent continua; received by the editors September 15, 1949.

(1) Amer. Math. Soc. Colloquium Publications, vol. 13, 1932. This book will be referred to as Foundations.

(2) A continuum which is irreducible between some two of its points will be said to be irreducible. The expression "an irreducible continuum $P Q$," or when no confusion results "a continuum $P Q$," will be used to mean a continuum which is irreducible between points $P$ and $Q$. 
THEOREM 1.2. In order that the continuum $M$ be atriodic and hereditarily unicoherent, it is necessary and sufficient that (1) if $A$ and $B$ are two points of $M$ there is not more than one subcontinuum $A B$ of $M$, and (2) every nondegenerate subcontinuum of $M$ is irreducible.

The condition is clearly sufficient. That it is necessary follows from a theorem proved by R. H. Sorgenfrey $\left({ }^{3}\right)$.

The following example shows that the second part of the condition is not a sufficient one that $M$ be unicoherent.

EXAmple. Knaster has shown that there exists a bounded plane continuum which is hereditarily indecomposable $\left({ }^{4}\right)$. Let $H$ and $K$ denote two such continua which have in common only two points $P$ and $Q, H$ and $K$ each being irreducible from $P$ to $Q$. The continuum $M$, which is the sum of $H$ and $K$, is not unicoherent, but every subcontinuum is irreducible.

Theorem 1.3. If a hereditarily irreducible continuum is the sum of two continua $H$ and $K$ whose common part is the sum of two mutually separated sets $U$ and $V$, then $H$ and $K$ contain indecomposable continua whose common part is the sum of two mutually separated sets lying in $U$ and $V$, respectively.

Proof. The continua $H$ and $K$ contain continua $H^{\prime}$ and $K^{\prime}$, respectively, such that $H^{\prime}$ is irreducible from $U$ to $V$ and $K^{\prime}$ from $H^{\prime} \cdot U$ to $H^{\prime} \cdot V$. Then $H^{\prime} \cdot K^{\prime}$ is the sum of two mutually separated sets $U^{\prime}$ and $V^{\prime}$ lying in $U$ and $V$, respectively, and $H^{\prime}$ and $K^{\prime}$ are each irreducible from $U^{\prime}$ to $V^{\prime}$. Suppose that $H^{\prime}$ is the sum of two continua $W$ and $Z$ distinct from itself. One of them, say $W$, contains no point of $V^{\prime}$ and the other no point of $U^{\prime}$, and $W \cdot Z$ contains no point of $K^{\prime}$. Since $W \cdot K^{\prime}, Z \cdot K^{\prime}$, and $W \cdot Z$ are mutually exclusive closed sets, no one of the continua $W, Z, K^{\prime}$ is a subset of the sum of the other two, and the continuum $H^{\prime}+K^{\prime}$ is not irreducible, contrary to hypothesis. Hence $H^{\prime}$ and $K^{\prime}$ are indecomposable.

COROLlaRy. In order that a continuum every subGontinuum of which is decomposable be atriodic and hereditarily unicoherent, it is necessary and sufficient that it be hereditarily irreducible.

THEOREM 1.4. In order that the continuum $M$ be atriodic and hereditarily unicoherent, it is necessary and sufficient that of any three points of $M$ there is one which weakly separates $\left(^{5}\right)$ the other two from each other in $M$.

THEOREM 1.5. If the atriodic unicoherent continuum $M$ is the sum of two

$\left(^{3}\right)$ R. H. Sorgenfrey, Concerning triodic continua, Amer. J. Math. vol. 66 (1944) pp. 439460. Cf. Theorem 3.2.

(4) B. Knaster, Un continu dont tout sous-continu est indecomposable, Fund. Math. vol. 3 (1922) pp. 245-286.

(5) If $A, B$, and $C$ are three points of a continuum $M$ such that every subcontinuum of $M$ which contains $A$ and $B$ contains $C$, then it is said that $C$ weakly separates $A$ from $B$ in $M$. 
continua $H$ and $K$ distinct from $M$, then $H$ and $K$ are unicoherent, and if $N$ is a non-unicoherent subcontinuum of $M$ intersecting $H$, it is a subset of $H\left(^{6}\right)$

Proof. Suppose that $K$ is the sum of two continua $U$ and $V$ such that $U \cdot V$ is the sum of two mutually exclusive closed sets $X$ and $Y$. The continuum $M$ is irreducible $\left({ }^{7}\right)$ between two points $A$ and $B$. One of these points, say $A$, belongs to $H-H \cdot K$ and the other to $K-H \cdot K$. The point $B$ belongs to $U$ or to $V$, say to $U$. If $H$ and $U$ had no common point, then $V$ and $H$ would intersect and $M$ would be the sum of the two continua $H+V$ and $U$, whose common part is $X+Y$, contrary to hypothesis. Hence $H+U$ is a continuum containing $A$ and $B$, and $M=H+U$. Since $V$ is not a subset of $U$, it follows that $V$ intersects $H$. Suppose $B$ is not in $V$. Then $H+V$ is a proper subcontinuum of $M$ and hence $(H+V) \cdot U$ is a continuum. There exist two open sets $D_{\boldsymbol{X}}$ and $D_{Y}$ containing $X$ and $Y$, respectively, such that $\bar{D}_{\boldsymbol{X}}$ and $\bar{D}_{Y}$ are mutually exclusive. Let $P$ and $Q$ denote points of $X$ and $Y$, respectively, and let $C_{P}$ and $C_{Q}$ denote the components of $V \cdot \bar{D}_{\mathbf{X}}$ and $V \cdot \bar{D}_{Y}$ concontaining $P$ and $Q$, respectively. By Theorem 39 of Chapter I of Foundations, neither $C_{P}$ nor $C_{Q}$ is a subset of $U$. Let $W$ and $Z$ denote the sets $C_{P}+(H+V) \cdot U$ and $C_{Q}+(H+V) \cdot U$, respectively. Consider the continua $U, W$, and $Z$. Since $C_{P}$ and $C_{Q}$ are subsets of $V$ it is clear that $(H+V) \cdot U$ $=U \cdot V=U \cdot Z=W \cdot Z$. The point $B$ is in $U$, but as $B$ is not in $V$, neither $W$ nor $Z$ contains $B$. Since $C_{P}$ and $C_{Q}$ are mutually exclusive and neither is a subset of $U, U \cdot W$ is a proper subcontinuum of $U, W$, and $Z$, which is impossible since $M$ is atriodic. Then $V$ contains $B$. By Theorem 115 of Chapter I of Foundations, $\subseteq(M-H)$ is connected $\left({ }^{8}\right)$. Since $M$ is irreducible from $A$ to $B$ and $B$ is in $U \cdot V$, it follows that $M-H=U-U \cdot H=V-V \cdot H$. The continuum $\mathfrak{S}(M-H)$, being a subset of $U \cdot V$, is a subset of $X$ or of $Y$, and therefore neither $U$ nor $V$ is a subset of $\subseteq(M-H)$. Since $M=U+H=V+H$ and $M$ is unicoherent, $H \cdot U$ and $H \cdot V$ are continua. Since $H \cdot U \cdot V$ contains $\mathfrak{S}(M-H) \cdot H$, there is a point common to the three continua $H \cdot U, H \cdot V$, and $\mathfrak{\complement}(M-H)$. The point $B$ is in $\mathbb{}(M-H)$ but in neither $H \cdot U$ nor $H \cdot V$. Since $U-U \cdot H=V-V \cdot H$, neither of the continua $H \cdot U, H \cdot V$ is a subset of the other. As neither $U$ nor $V$ is a subset of $\mathfrak{E}(M-H), \mathfrak{S}(M-H)$ contains neither $H \cdot U$ nor $H \cdot V$. Thus the three continua $H \cdot U, H \cdot V$, and $\mathfrak{S}(M-H)$ have a common point and no one is a subset of the sum of the other two. But, by a theorem proved by R. H. Sorgenfrey $\left({ }^{9}\right)$, this is contrary to the hypothesis that $M$ is atriodic. From this contradiction, it follows that $K$, and similarly $H$, is unicoherent.

Suppose that $N$ is not a subset of $H$. If $H$ is not a subset of $N$, it follows

(6) The first part of the conclusion was suggested by R. L. Swain.

( ${ }^{7}$ Sorgenfrey, loc. cit.

$\left.{ }^{8}\right)$ For typographical convenience, $\mathfrak{S}(X)$ is sometimes used instead of $\bar{X}$ for the closure of $X$.

(9) Ibid., cf. Theorem 1.8 . 
from the first part of this theorem that $H+N$ is not unicoherent. If $H$ is a subset of $N$, then $H+N=N$ and $H+N$ is not unicoherent. Since $M$ is unicoherent, $H+N$ is a proper subset of $M$. Then $M=(H+N)+K$, which is contrary to the first part of the theorem.

THEOREM 1.6. If $M$ is an atriodic unicoherent continuum and $N$ is a nonunicoherent subcontinuum of $M$, then $N$ is a continuum of condensation ${ }^{10}$ ) of $M$.

Proof. Let $A$ and $B$ denote points such that $M$ is irreducible from $A$ to $B$. If $N$ contains $A$ or $B$, then $M$ is the sum of the continua $N$ and $\mathfrak{S}(N-H)$, and it follows from Theorem 1.5 that $M=\mathfrak{S}(N-H)$ and hence that $N$ is a continuum of condensation of $M$. Suppose $N$ contains neither $A$ nor $B$. If $M-N$ is connected, then $\subseteq(M-N)$ is a continuum containing $A$ and $B$ and hence every point of $N$ is a limit point of $M-N$. If $M-N$ is not connected, let $U$ and $V$ denote the two components of $M-N$ which contain $A$ and $B$, respectively. Since $M$ is irreducible from $A$ to $B, M$ is the sum of the two continua $\bar{U}$ and $V+N$. Then, by Theorem $1.5, \bar{U}$ contains $N$, and $N$ is therefore a continuum of condensation of $M$.

THEOREM 1.7. If $A B$ is an irreducible continuum, every non-unicoherent subcontinuum of $A B$ contains a non-unicoherent subcontinuum which is a continuum of condensation of $A B$.

Proof. Consider $A B$ as space. If $A B$ contains a non-unicoherent continuum $L$, there is a subcontinuum $N$ of $L$ which is the sum of two continua $H$ and $K$ such that $H \cdot K$ is the sum of two mutually separated sets $X$ and $Y$, $H$ and $K$ each being irreducible from $X$ to $Y$. If $N$ contains $A$, say that $A$ belongs to $H$. The continuum $\mathbb{S}(A B-N)$ intersects $H$, for otherwise $A B$ would not be unicoherent. The boundary of $A B-N$ contains $K-H \cdot K$, and therefore, since $K$ is irreducible from $X$ to $Y$, it contains $K$. As $A B$ is unicoherent, $H \cdot \mathfrak{C}(A B-N)$ is a continuum lying in $H$, and since it contains $X$ and $Y$, it is $H$. Then the boundary of $A B-N$ contains $H$. Hence if $N$ contains $A$ or $B$ it is a continuum of condensation. Suppose that $N$ contains neither $A$ nor $B$. If $A B-N$ is connected, every point of $N$ is a limit point of $A B-N$. If $A B$ is not connected, let $U$ and $V$ denote the components of $A B-N$ containing $A$ and $B$, respectively. Suppose that neither $H$ nor $K$ intersects both $\bar{U}$ and $\bar{V}$; say that $H$ intersects $\bar{U}$. Then $A B$ is the sum of the continua $U+H$ and $V+K$, the common part of which is not connected, contrary to hypothesis. Hence one of the sets $H, K$, say $H$, intersects both $\bar{U}$ and $\bar{V}$. Then since $A B=\bar{U}+H+\bar{V}$, every point of $K-H \cdot K$, and therefore every point of $K$, is a limit point of $U+V$. If $\bar{U}$ and $\bar{V}$ do not intersect, then one of the sets $\bar{U}, \bar{V}$, say $\bar{U}$, contains $K$ and the other no point of $K$. But then

(10) A subcontinuum $N$ of a continuum $M$ is said to be a continuum of condensation of $M$ if every point of $N$ is a limit point of $M-N$. 
$M$ is the sum of two continua $\bar{U}$ and $H+V$, the common part of which is not connected. Hence $\bar{U}$ intersects $\bar{V}, A B=\bar{U}+\bar{V}$, and $N$ is a continuum of condensation.

\section{Properties implying hereditary unicoherence.}

THEOREM 2.1. If $M$ is a continuum such that of any four points of $M$ there is one which weakly separates two of the others from each other in $M$, then $M$ is hereditarily unicoherent.

If $M$ is a continuum having the property that for some positive integer $k$ greater than four it is true that of every $k$ points of $M$ there is one which weakly separates two of the others from each other in $M$, it does not follow that $M$ is unicoherent, as can be seen by considering the continuum $M$ of the example following Theorem 1.2 .

It will be shown in Theorem 2.8 , however, that if the stipulation that $M$ be hereditarily decomposable is added to the hypothesis of Theorem 2.1 , then "four" can be replaced by " $k$," where $k$ is any positive integer greater than two.

LemмA A. If the irreducible continuum $M$ is hereditarily decomposable, it contains two, and only two, mutually exclusive continua $H$ and $K$ such that (1) in order that $M$ be irreducible between two of its points it is necessary and sufficient that one of them belong to $H$ and the other to $K$, (2) $M-H$ and $M-(H+K)$ are strongly connected $\left({ }^{11}\right)$, (3) $H$ is a subset of every subcontinuum of $M$ which intersects both $H$ and $M-H$, and (4) if $N$ is a proper subcontinuum of $M$ which intersects both $H$ and $M-H$, then every subcontinuum of $M$ which intersects $N$ and $K$ contains $\subseteq(M-N)$, and $\mathbb{S}(M-N)$ is irreducible from $N$ to $K$.

Proof. Let $A$ and $B$ denote points between which $M$ is irreducible. Let $H$ denote the set of all points $x$ such that $M$ is irreducible from $B$ to $x$, and $K$ the set of all points $x$ such that $M$ is irreducible from $A$ to $x$. The sets $H$ and $K$ are mutually exclusive continua, and $M$ is irreducible from $H$ to $K$. Let $P$ and $Q$ be two points of $M-H$. Since $M$ is not irreducible from $B$ either to $P$ or to $Q$, there exist continua lying in $M-H$ and containing $B+P$ and $B+Q$, respectively. Hence $M-H$ is strongly connected, and therefore if $M$ is irreducible between two points, one of them belongs to $H$ and the other to $K$.

Suppose there are two points $P$ and $Q$ of $M-(H+K)$ such that no continuum containing $P$ and $Q$ lies in $M-(H+K)$. There exist two continua $X$ and $Y$, each irreducible from $P$ to $Q$, lying in $M-K$ and $M-H$, respectively. Since $X$ and $Y$ intersect $H$ and $K$, respectively, $M=X+Y$. Then $H$ is a subset of $X-X \cdot Y$, and $K$ of $Y-X \cdot Y$. Suppose there exists a proper

(11) A set $M$ is said to be strongly connected if each two points of $M$ are in some continuum lying in $M$. 
subcontinuum $W$ of $X$ containing $A$ and $P$ and a proper subcontinuum $Z$ of $Y$ containing $B$ and $P$. But neither $W$ nor $Z$ contains $Q$ and $W+Z$ is then a continuum containing $A$ and $B$. From this contradiction it follows that either $X$ is irreducible from $A$ to $P$ or $Y$ is irreducible from $B$ to $P$; say the former. Let $T$ denote the set of all points $x$ such that $X$ is irreducible from $P$ to $x$. The set $T$ is a continuum, and since $T$ contains $A$ and $Q, M=T+Y$. Therefore $X-T$ is a subset of $Y$, and, by Theorem 106 of Chapter I of Foundations, $X$ is a subset of $Y$. But $X$ contains $H$ and $Y$ does not. From this contradiction it follows that $M-(H+K)$ is strongly connected.

Suppose that $M$ contains a continuum $W$ which intersects $H$ and $M-H$ but does not contain $H$. There exists a continuum $Z$ which lies in $M-H$ and intersects $W$ and $K$. But $W+Z$ would be a proper subcontinuum of $M$ intersecting $H$ and $K$.

Let $N$ be a proper subcontinuum of $M$ intersecting $H$ and $M-H$. Suppose there exists a subcontinuum $L$ of $M$ which intersects $N$ and $K$ but does not contain $M-N$. Then $N+L$ is a proper subcontinuum of $M$ intersecting $H$ and $K$. From this contradiction, (4) follows.

Definitron. If $M$ is an irreducible hereditarily decomposable continuum, the subcontinua $H$ and $K$ of $M$ described in Lemma $\mathrm{A}$ will be called the $E$-subcontinua of $M$.

Lемма B. If $M$ is an irreducible continuum which is hereditarily decomposable, $H$ and $K$ are the E-subcontinua of $M$, and $P$ is a point of $M-(H+K)$, then there exists a continuum $T$ containing $P$ and lying in $M-(H+K)$ such that $M-T$ is the sum of two mutually separated connected sets $U$ and $V$ containing $H$ and $K$, respectively, such that (1) $M=\bar{U}+\bar{V}$, (2) $\bar{U}$ is irreducible from $H$ to $\bar{V}$, and $\bar{V}$ is irreducible from $K$ to $\bar{U}$, (3) the E-subcontinua of $\bar{U}$ are $H$ and $T \cdot \bar{U}$ and the E-subcontinua of $\bar{V}$ are $K$ and $T \cdot \bar{V}$, and (4) every subcontinuum of $M$ which intersects $H$ and $T+V$ contains $\bar{U}$.

Proof. There exists a proper subcontinuum $N$ of $M$ which is irreducible from $H$ to $P$. Let $Z$ denote $M-N$. By Lemma A, $\bar{Z}$ is a proper subcontinuum of $M$ irreducible from $N$ to $K$. Let $W$ denote $M-\bar{Z}$. Then $\bar{W}$ is irreducible from $H$ to $\bar{Z}, \bar{Z}$ is irreducible from $\bar{W}$ to $K$, and $M=\bar{W}+\bar{Z}$. It is clear that $H$ is one of the $E$-subcontinua of $\bar{W}$; let $K^{\prime}$ denote the other. Similarly $K$ is one of the $E$-subcontinua of $\bar{Z}$; let $H^{\prime}$ denote the other. Since $\bar{W} \cdot \bar{Z}$ is a subset of $H^{\prime}$ and of $K^{\prime}, H^{\prime}+K^{\prime}$ is a continuum. Let $T=H^{\prime}+K^{\prime}$. If $P$ belongs to $\bar{W}$, then since $\bar{W}$ is a subset of $N$ and $N$ is irreducible from $H$ to $P, P$ belongs to $K^{\prime}$. If $P$ belongs to $\bar{Z}$, then since $\bar{Z}$ is irreducible from $K$ to $N, P$ belongs to $H^{\prime}$. Hence $T$ contains $P$. Let $U=\bar{W}-K^{\prime}$ and $V=\bar{Z}-H^{\prime}$. Then $U$ and $V$ are connected sets containing $H$ and $K$, respectively, such that $\bar{W}=\bar{U}$ and $\bar{Z}=\bar{V}$. Let $L$ be a subcontinuum of $M$ which intersects both $H$ and $T+V$. It follows from Lemma A that if $L$ intersects $T$ or $V$ it intersects $\bar{V}$ and hence contains $\bar{U}$. 
Definition. If $M$ is an irreducible hereditarily decomposable continuum and $T$ is a subcontinuum of $M$ having the properties described in Lemma $\mathrm{B}$, $T$ will be called a $C$-subcontinuum of $M$.

Lemma C. The C-subcontinua of an irreducible hereditarily decomposable continuum $M$ are mutually exclusive.

Proof. Suppose the $C$-subcontinua $T_{1}$ and $T_{2}$ of $M$ have a point $P$ in common and that $T_{2}$ contains a point $Q$ not in $T_{1}$. Let $H$ and $K$ be the $E$-subcontinua of $M$. The set $M-T_{i}, i=1,2$, is the sum of two mutually separated connected sets $U_{i}$ and $V_{i}$ containing $H$ and $K$, respectively. Either $U_{1}$ or $V_{1}$ contains $Q$; say the former. By Lemma A, $U_{1}$ contains a continuum which contains $Q$ and intersects $H$, and therefore, by Lemma $\mathrm{B}, U_{1}$ contains $\bar{U}_{2}$. The continuum $T_{1}+V_{1}$ contains $P$, and therefore, by Lemma $\mathrm{B}$, it contains $\bar{V}_{2}$. But $U_{1}$ and $T_{1}+V_{1}$ are mutually exclusive, while $\bar{U}_{2}$ and $\bar{V}_{2}$ intersect. From this contradiction the lemma follows.

THEOREM 2.2. If the hereditarily decomposable continuum $M$ is irreducible from point $A$ to point $B$, and $g_{A}$ and $g_{B}$ are the E-subcontinua of $M$ containing $A$ and $B$, respectively, and $G$ is the collection consisting of $g_{A}, g_{B}$, and all the $C$-subcontinua of $M$, then $G$ is an upper semi-continuous collection of mutually exclusive continua filling $u p M$, and $M$ is an arc from $g_{A}$ to $g_{B}$ with respect to the elements of $G$.

Proof. It follows from Lemmas A, B, and $\mathrm{C}$ that $G$ is a collection of mutually exclusive continua filling up $M$. Let $g$ be any element of $G$ distinct from $g_{A}$ and $g_{B}$. By Lemma B, $M-g$ is the sum of two mutually separated connected sets $U$ and $V$ containing $g_{A}$ and $g_{B}$, respectively. Then $g$ separates $g_{A}$ from $g_{B}$ in $M$. Let $P$ be a point of $U-g_{A}$, and let $g_{P}$ denote the element of $G$ containing $P$. Since $g_{P}$ is a connected subset of $M-g$, it is a subset of $U$. By Lemma B, $M-g_{P}$ is the sum of two mutually separated connected sets $U_{P}$ and $V_{P}$ containing $g_{A}$ and $g_{B}$, respectively. The continuum $g+V$ is a connected subset of $M-g_{P}$ and hence is a subset of $V_{P}$. Then $g_{P}$ separates $g$ from $g_{A}$ in $M$. Similarly there is an element of $G$ which separates $g$ from $g_{B}$ in $M$. Let $g_{1}$ and $g_{2}$ be two elements of $G$ distinct from $g_{A}$ and $g_{B}$. For each $i, i=1,2$, $M-g_{i}$ is the sum of two mutually separated connected sets $U_{i}$ and $V_{i}$ containing $g_{A}$ and $g_{B}$, respectively. The continuum $g_{1}$ is a subset of $U_{2}$ or of $V_{2}$, say of $U_{2}$. Then $g_{2}+V_{2}$ is a connected subset of $M-g_{1}$ and hence is a subset of $V_{1}$. Since $V_{1}$ contains a point not in $g_{2}+V_{2}$, it intersects $U_{2}$. Let $Q$ be a point of $U_{2} \cdot V_{1}$, and let $g_{Q}$ be the element of $G$ containing $Q$. The set $g_{Q}$ is a subset of $U_{2} \cdot V_{1}$. The set $M-g_{Q}$ is the sum of two mutually separated connected sets $U_{Q}$ and $V_{Q}$ containing $g_{A}$ and $g_{B}$, respectively. Since $g_{1}+U_{1}$ is a connected subset of $M-g_{Q}$, it is a subset of $U_{Q}$; similarly, $g_{2}+V_{2}$ is a subset of $V_{Q}$. Therefore any two elements of $G$ are separated from each other in $M$ by some element of $G$. It follows from Theorem $40, \S 1$, of Chapter $\mathrm{V}$ of 
Foundations that $G$ is an upper semi-continuous collection, and that $M$ is an acyclic continuous curve with respect to the elements of $G$. By Theorem 6, $\S 1$, of the same chapter, it follows that the "acyclic continuous curve" $G$ is compact, and since every element of $G$ distinct from $g_{A}$ and $g_{B}$ separates $M$, $M$ is an arc from $g_{A}$ and $g_{B}$ with respect to the elements of $G$.

Theorem 2.3. The elements of the collection $G$ described in Theorem 2.2 are the dendratomic subsets of $M$.

Proof. By a theorem due to R. L. Moore $\left({ }^{12}\right)$, every compact irreducible continuum has dendratomic subsets. Moore $\left({ }^{13}\right)$ has also shown that if $P$ is a point of $M$, the dendratomic subset of $M$ containing $P$ is the set of all points $x$ such that there do not exist uncountably many mutually exclusive subcontinua of $M$ each separating $P$ from $x$ in $M$. Suppose there exists a subcontinuum $N$ of $M$ which separates two points $P$ and $Q$ of $g_{A}$ from each other in $M$. The set $N$ intersects $g_{A}$. It does not intersect $M-g_{A}$, for, by Lemma A, it would then contain $g_{A}$. Hence $N$ is a subset of $g_{A}$. But by Theorem 115 of Chapter I of Foundations, $M-N$ would then be connected. Hence no subcontinuum of $M$ separates two points of $g_{A}$ or two points of $g_{B}$ from each other in $M$. Let $g$ be any element of $G$ distinct from $g_{A}$ and $g_{B}$. Let $N$ be a subcontinuum of $M$ which separates two points $P$ and $Q$ of $g$ from each other in $M$. The set $M-g$ is the sum of two mutually separated connected sets $U$ and $V$ having the properties described in Lemma B. Suppose that $P$ and $Q$ belong to $\bar{U}$. Then $N$ intersects the continuum $\bar{U}$. Suppose that $N$ intersects $U$; let $C$ be a component of $N-N \cdot g$ which intersects $U$. The continuum $\bar{C}$ contains a point of $g \cdot \bar{U}$, and by Lemma B, $N$ contains $g \cdot \bar{U}$. But $N$ does not contain $P$ or $Q$. Hence $N$ is a subset of $g+\bar{V}$ and therefore every point of $N \cdot \bar{U}$ is a limit point of $U$. Hence $\bar{U}-N \cdot \bar{U}$ is connected and it contains both $P$ and $Q$. From this contradiction it follows that $P$ and $Q$ do not both belong to $\bar{U}$, and similarly they do not belong to $\bar{V}$. Then one of them belongs to $g-g \cdot \bar{U}$ and the other to $g-g \cdot \bar{V}$. It can be shown as before that $N$ is a subset of $g$. Suppose there is a point $O$ of $\bar{U} \cdot \bar{V}$ which is not in $N$. The set $U+V+O$ $+P+Q$ is a connected subset of $M-N$ and $N$ does not separate $P$ from $Q$ in $M$. Therefore $N$ must contain $\bar{U} \cdot \bar{V}$. Since every subcontinuum of $M$ which separates $P$ from $Q$ in $M$ contains $\bar{U} \cdot \bar{V}$, there do not exist two mutually exclusive subcontinua of $M$ which separate $P$ from $Q$ in $M$. If $P$ and $Q$ are points belonging to different elements of $G$, then, since $G$ is an arc with respect to its elements, there exist uncountably many mutually exclusive subcontinua of $M$ which separate $P$ from $Q$ in $M$. Hence the elements of $G$ are the dendratomic subsets of $M$.

THEOREM 2.4. If the elements of collection $G$ described in Theorem 2.2 are

(12) Bull. Amer. Math. Soc. vol. 47 (1941). Cf. p. 866.

(13) R. L. Moore, On the structure of continua, Rice Institute Pamphlet vol. 23 (1936) pp. 58-74, cf. p. 73. 
unicoherent, so is $M$.

Proof. Let $W$ be any subcontinuum of $M$ which intersects $g_{A}$ but not $g_{B}$. Since $W$ is closed and $G$ is an "arc" from $g_{A}$ to $g_{B}$, there is a last element of $G$, in the order from $g_{A}$ to $g_{B}$, which intersects $W$. Let $w$ denote this element. The set $M-w$ is the sum of two mutually separated connected sets $U$ and $V$ such that all the elements of $G$ which precede $w$ in the order from $g_{A}$ to $g_{B}$ are subsets of $U$. By Lemma $\mathrm{B}, W$ contains $\bar{U}$ and $\bar{U} \cdot w$ is a continuum. Suppose that $W \cdot w$ is the sum of two mutually separated sets, $X$ and $Y$. The continuum $\bar{U} \cdot w$ is a subset of $X$ or of $Y$, say of $X$. Then since $W$ is a subset of $U+w, W$ is the sum of two mutually exclusive closed sets, $U+X$ and $Y$. From this contradiction it follows that $W \cdot w$ is a continuum. Let $W$ and $Z$ be proper intersecting subcontinua of $M$ intersecting $g_{A}$ and $g_{B}$, respectively. Let $w$ be the last element of $G$, in the order from $g_{A}$ to $g_{B}$, which intersects $W$ and let $z$ be the first element which intersects $Z$. If $w$ and $z$ are distinct, $z$ precedes $w$ on $G$ in the order from $g_{A}$ to $g_{B}$. Clearly every element of $g$ which lies between $w$ and $z$ is a subset of $W \cdot Z$, and $W \cdot Z$ is the sum of these elements and the continua $W \cdot w$ and $Z \cdot z$ and hence is connected. If $w=z, W \cdot Z$ is a subset of $w$. But $W \cdot w+Z \cdot w=w$, and since $W \cdot w$ and $Z \cdot w$ are continua and $w$ is unicoherent, so is $M$.

Theorem 2.5. If the collection $G$ described in Theorem 2.2 is such that if $g$ is an element of $G$, and $U$ and $V$ are the two mutually separated sets whose sum is $M-g$, then $g$ is a subset of $\bar{U}$ and $\bar{V}$; in particular if $G$ is continuous, it follows that $M$ is unicoherent,

Proof. Suppose on the contrary that $M$ is the sum of two continua $L$ and $N$ the common part of which is not conmected. Since $M$ is irreducible from $g_{A}$ to $g_{B}$, one of the sets $g_{A}, g_{B}$ is a subset of $M-L$ and the other of $M-N$; say that $g_{A}$ is a subset of $M-N$. Let $P$ be a point of $L$ which does not belong to $g_{A}$. Let $g_{P}$ denote the element of $G$ which contains $P$. Clearly $L$ contains every element of $G$ which precedes $g_{P}$ in the order from $g_{A}$ to $g_{B}$ on the "arc" $G$. The set $M-g_{P}$ is the sum of two mutually separated sets $U$ and $V$, and $L$ contains $\bar{U}$ and hence $g_{P}$. Thus $L$ is the sum of all the continua of $G$ which intersect it, and so is $N$. But $G$ is unicoherent with respect to its elements. Hence $M$ is unicoherent.

THEOREM 2.6. If $M$ is a hereditarily decomposable continuum which is not unicoherent, then $M$ contains a continuum $N$ which is a simple closed curve with respect to the elements of an upper semi-continuous collection $G$ of mutually exclusive continua filling up $N$; the collection $G$ is such that if $h$ and $k$ are any two of its elements, then $N-(h+k)$ is the sum of two mutually separated connected sets $U$ and $V$ such that (1) $N=\bar{U}+\bar{V}$, (2) each of the continua $\bar{U}, \bar{V}$ is irreducible from $h$ to $k$, and (3) any subcontinuum of $h+U+k$ which intersects $h$ and $k$ contains $\bar{U}$. 
Proof. Since $M$ is not unicoherent, there are subcontinua $L$ and $N$ of $M$ such that $L \cdot N$ is the sum of two mutually separated sets $X$ and $Y, L$ and $N$ each being irreducible from $X$ to $Y$. One of the $E$-subcontinua of $L$ contains $X$ and the other contains $Y$, and the same is true for $N$. Let $H_{L}$ and $H_{N}$ denote the $E$-subcontinua of $L$ and $N$, respectively, which contain $X$, and $K_{L}$ and $K_{N}$ those which contain $Y$. Let $H=H_{L}+H_{N}$ and $K=K_{L}+K_{N}$. Let $G_{L}^{\prime}$ denote the collection consisting of $H, K$, and all the $C$-subcontinua of $L$, and let $G_{N}^{\prime}$ be a similar collection for $N$. It is clear that (1) $G_{L}^{\prime}$ is an upper semicontinuous collection of mutually exclusive continua filling up $H+L+K$ and that, with respect to its elements, it is an arc from $H$ to $K$, and (2) $G_{N}^{\prime}$ is an upper semi-continuous collection of mutually exclusive continua filling up $H+N+K$ and that, with respect to its elements, it is an arc from $H$ to $K$. Let $G$ denote the collection $G_{L}^{\prime}+G_{N}^{\prime}$. Then $G$ satisfies all the stipulated requirements.

THEOREM 2.7. In order that a hereditarily decomposable continuum be hereditarily unicoherent, it is necessary and sufficient that it contain no continuum $N$ such that $N$ is a simple closed curve with respect to the elements of some upper semi-continuous collection of mutually exclusive continua filling up $N$.

Theorem 2.7 is a consequence of the preceding theorem and Theorems 4 and $5, \S 1$, of Chapter V of Foundations.

THEOREM 2.8. If $M$ is a hereditarily decomposable continuum which has the property that for some positive integer $k$ it is true that of every $k$ points of $M$ there is one which weakly separates two of the others from each other in $M$, then $M$ is hereditarily unicoherent.

Proof. Suppose that $M$ is not hereditarily unicoherent. By Theorem 2.6, $M$ contains a continuum $N$ which is a simple closed curve with respect to the elements of an upper semi-continuous collection $G$ of mutually exclusive continua filling up $N$. Let $g_{1}, g_{2}, g_{3}, \cdots, g_{k}$ be distinct elements of $G$ which lie on this "simple closed curve" in the order $g_{1}, g_{2}, g_{3}, \cdots, g_{k}, g_{1}$. For each positive integer $n$ not exceeding $k$, let $P_{n}$ denote a point of $g_{n}$. Let $g_{0}$ denote $g_{k}$, and let $g_{k+1}$ denote $g_{1}$. For each positive integer $n$ not exceeding $k$, the "simple closed curve" $G$ is the sum of two "arcs" $g_{n-1} g_{n+1}$ having in common only their end "points." In each case one of these "arcs" contains $g_{i}$ for every $i \neq n$ but does not contain $g_{n}$. By Theorems 4 and 5, $\S 1$, of Chapter V of Foundations, the sum of the elements of $G$ which are "points" of this arc is a continuum. This continuum does not contain $P_{n}$ but contains all the points $P_{i}$ $i \neq n$. But this is contrary to the hypothesis.

THEOREM 2.9. If $M$ is a hereditarily decomposable continuum every subcontinuum of which is irreducible about a closed proper subset having only countably many components, then $M$ is hereditarily unicoherent. 
Proof. Suppose that $M$ is not hereditarily unicoherent. By Theorem 2.6, $M$ contains a continuum $N$ which is a simple closed curve with respect to the elements of an upper semi-continuous collection $G$ of mutually exclusive continua filling up $N$, the collection $G$ having the properties described in the theorem. By hypothesis, $N$ is irreducible about a closed proper subset $H$ which has only countably many components. Suppose some component $K$ of $H$ intersects every element of $G$. Let $P$ be a point of $N-K$. Let $p$ be the element of $G$ which contains $P$, and let $h$ and $k$ be two other elements of $G$. Then $N-(h+k)$ is the sum of two mutually separated connected sets $U$ and $V, U$ containing $p$. The set $N-(h+p)$ is the sum of two mutually separated sets $U_{1}$ and $V_{1}, V_{1}$ containing $k$, and the set $N-(p+k)$ is the sum of two mutually separated sets $U_{2}$ and $V_{2}, V_{2}$ containing $h$. It follows that $U+h+k$ is the sum of the elements of $G$ which belong to the "arc" $h p k$ of the "simple closed curve" $G$ and that $(U+h+k)-p$ is the sum of the two mutually separated connected sets $h+U_{1}$ and $k+U_{2}$, and also that $\bar{U}=\bar{U}_{1}+\bar{U}_{2}$. Let $C$ be a component of $(U+h+k) \cdot K$ which contains a point of $p$. The continuum $C$ contains a point of $h+k$, but does not intersect both $h$ and $k$, since in that case it would contain $\bar{U}$ and therefore $P$. Say that $C$ does not intersect $h$. It follows that $C$ contains $\bar{U}_{2}$ and hence contains every element of $G$ which lies between $p$ and $k$ on the "arc" $h p k$ of the "simple closed curve" G. It can likewise be shown that if any component of $(U+h+k) \cdot K$ intersected both $U_{1}$ and $k$, it would contain $P$. Hence no component of $(U+h+k) \cdot K$ intersects both $U_{1}$ and $k$. Let $a$ and $b$ be two elements of $G$ which are subsets of $U_{1}$, $a$ preceding $b$ on the "arc" $h p k$ of the "simple closed curve" $G$ in the order from $h$ to $k$. Let $B$ be a point of $b \cdot K$ and $W$ be the component of $(U+h+k) \cdot K$ which contains $B$. Then $W$ contains a point of $h$, and it can be shown that $W$ contains $a$. Hence $\bar{U}_{1}$ is a subset of $K$. But $\bar{U}=\bar{U}_{1}+\bar{U}_{2}$, and hence $\bar{U}$ is a subset of $K$ and $K$ contains $P$. From this contradiction it follows that no component of $H$ intersects every element of $G$.

If $K$ is any subset of $N$, let $G_{K}$ denote the collection of all elements of $G$ that intersect $K$. For each component $K$ of $H, G_{K}$ is a proper subcollection of $G$ and therefore $G_{K}^{*}\left({ }^{14}\right)$ is a proper subset of $N$. By Theorem 2, $\S 1$, of Chapter V of Foundations, $G_{K}^{*}$ is closed. Since $K$ is connected, so is $G_{K}^{*}$. Furthermore, if $K$ is not a subset of an element of $G, G_{K}$ is an "arc" with respect to its elements. In this case, let $h$ and $k$ denote the elements of $G_{K}$ which are the end "points" of this "arc." Then $N-(h+k)$ is the sum of two mutually separated connected sets $U$ and $V$, and $U+h+k=G_{K}^{*}$. Since $K$ is a subcontinuum of $U+h+k$ intersecting $h$ and $k$, it follows that $\bar{U}$ is a subset of $K$. Then $K$ contains every element of $G_{K}$ except $h$ and $k$.

Suppose that $H$ has two components $X$ and $Y$ such that neither $X$ nor $Y$ is a subset of an element of $G$. Suppose that $G_{X}^{*}$ and $G_{Y}^{*}$ intersect. There exist. elements $h_{X}$ and $k_{X}$ of $G_{X}$ such that $G_{X}$ is an "arc" from $h_{X}$ to $k_{X}$ with respect

(14) If $G$ is a collection of point sets, $G^{*}$ denotes the sum of all the sets of $G$. 
to its elements, and likewise there exist elements $h_{Y}$ and $k_{Y}$ of $G_{Y}$ such that $G_{Y}$ is an "arc" from $h_{Y}$ to $k_{Y}$ with respect to its elements. Moreover, every element of $G_{X}$ except $h_{X}$ and $k_{X}$ is a subset of $X$, and every element of $G_{Y}$ except $h_{Y}$ and $k_{Y}$ is a subset of $Y$. Hence $G_{X}^{*}-\left(h_{X}+k_{X}\right)$ and $G_{Y}^{*}-\left(h_{Y}+k_{Y}\right)$ are mutually exclusive, and one of the elements $h_{X}, k_{X}$ is identical with one of the elements $h_{Y}, k_{Y}$; say that $k_{X}=h_{Y}$. Then by Theorem $2.6, k_{X}$ is a subset of $X+Y$, and therefore $X$ and $Y$ have a point in common. This involves a contradiction. Hence if $X$ and $Y$ are two components of $H$ such that neither is a subset of an element of $G$, and $Z$ is a component of $H$ which is a subset of an element $z$ of $G$, then $z$ is not in both $G_{X}$ and $G_{Y}$. Let $C_{1}, C_{2}, C_{3}, \cdots$ denote the components of $H$, and for each positive integer $n$, let $G_{n}$ denote the collection of all elements of $G$ which intersect $C_{n}$. It is clear from the results just stated that the set $L=G_{1}^{*}+G_{2}^{*}+G_{3}^{*}+\cdots$ is the sum of a countable number of mutually exclusive subcontinua of $N$, although $G_{1}^{*}, G_{2}^{*}, \cdots$ are not necessarily disjoint. By Theorem 2, $\$ 1$, of Chapter $\mathrm{V}$ of Foundations, $L$ is closed. Since $N$ is connected, it follows from a theorem of Sierpinski's( $\left.{ }^{15}\right)$ that $L$ is a proper subset of $N$. Then there exists a proper subcollection of $Q$ of $G$ such that $Q$ is an "arc" with respect to its elements and $Q^{*}$ contains $L$. Hence $Q^{*}$ is a proper subcontinuum of $N$ which contains $H$. But $N$ is irreducible about $H$. From this contradiction the theorem follows.

3. Properties of hereditarily unicoherent continua analogous to properties of an acyclic curve.

Definition. The point $P$ is said to be a terminal point of the continuum $M$ if every irreducible subcontinuum of $M$ which contains $P$ is irreducible from $P$ to some point.

If an irreducible subcontinuum, a terminal point, and a weak cut point $\left({ }^{16}\right)$ of a hereditarily unicoherent continuum $M$ are regarded as the analogues of an arc, an end point, and a cut point, respectively, $M$ has properties analogous to certain properties of an acyclic continuous curve, and if $M$ is hereditarily decomposable, it has more such properties.

THEOREM 3.1. Every point of a hereditarily unicoherent continuum $M$ is either a terminal point or a weak cut point of $M$, and if $P$ is a point of the subcontinuum $A B$ of $M$ distinct from $A$ and $B$, then $P$ weakly separates $A$ from $B$ in $M$.

The terminal points of a hereditarily unicoherent continuum do not play exactly the same role as the end points of a compact acyclic continuous curve. For instance the points $A$ and $B$ of Theorem 1.1 are not necessarily terminal points of the subcontinuum $A B$. The set of weak cut points and the set of terminal points are not necessarily mutually exclusive. A hereditarily uni-

(15) Cf. Foundations, Theorem 44 of Chapter I.

(16) The point $P$ of the continuum $M$ is said to be a weak cut point of $M$ if $M-P$ is not strongly-connected. 
coherent continuum $M$ does not necessarily have a terminal point, even if it is atriodic. It will be shown in Theorem 3.5, however, that if $M$ is hereditarily decomposable it has two terminal points.

R. L. Moore has proved $\left({ }^{17}\right)$ the following theorem for the plane by an argument which is valid in any metric space: If $M$ is a hereditarily unicoherent continuum and $H$ is an irreducible subcontinuum of $M$, then $H$ is a subset of an irreducible subcontinuum of $M$ which is not a subset of any other irreducible subcontinuum of $M$.

Definition. An irreducible subcontinuum of a continuum will be called a maximal one if it is not a subset of another one.

ThEOREM 3.2. If $M$ is a hereditarily unicoherent continuum, $A B$ is a maximal irreducible subcontinuum of $M$, and $P Q$ is an irreducible subcontinuum of $M$ which contains $A$ but is not irreducible from $A$ to any point, then $P Q$ is a subset of $A B$.

Proof. If neither $P$ nor $Q$ belongs to $A B$, then neither $P B$ nor $Q B$ contains $A$. For if $A$ were in $P B, A B$ would be a subset of $P B$ and would not be a maximal irreducible subcontinuum of $M$. Hence $P B$, and similarly $Q B$, fails to contain $A$. But this involves a contradiction since $P B+Q B=P Q$. Suppose that $A B$ contains $Q$ but not $P$. As before, $P B$ does not contain $A$. Then $Q B$ contains $A$ since $Q B+P B$ contains $P Q$, and therefore $A B=Q B$ and $P B$ does not contain $Q$ since in that case it would contain $A$. Since $P Q$ is not irreducible from $A$ to any point, $A P$ does not contain $Q$. Hence $A B$ contains both $P$ and $Q$ and therefore contains $P Q$.

CoRollary. In order that a point be a terminal point of a compact hereditarily unicoherent continuum $M$ it is necessary and sufficient that it be a terminal point of a maximal irreducible subcontinuum of $M$.

THEOREM 3.3. If $M$ is a hereditarily unicoherent continuum irreducible from point $A$ to point $B$ and $P Q$ is an irreducible subcontinuum of $M$ which contains $A$ but is not irreducible from $A$ to any point, then $M$ is irreducible from $B$ to each point of $P Q$.

Proof. Either $B P$ or $B Q$ contains $A$, since $B P+B Q$ contains $P Q$. Suppose that $B P$ contains $A$ but $B Q$ does not. Then $B Q$ does not contain $P$, since $M$ is irreducible from $B$ to $P$. Since $P Q$ is not irreducible from $A$ to any point, $A Q$ does not contain $P$. But $B Q+A Q=M$. Hence $B Q=B P=M$. Suppose there is a point $X$ of $P Q$ such that $B X$ is not $M$, in which case $B X$ contains neither $P$ nor $Q$. Hence $B X+A X$ does not contain both $P$ and $Q$. But $B X+A X=M$.

COROLlaRY 1. If $M$ is a hereditarily unicoherent continuum which is irre-

(17) R. L. Moore, Concerning compact continua which contain no continuum which separates the plane, Proc. Nat. Acad. Sci. U.S.A. vol. 29 (1934) pp. 41-45. 
ducible from point $A$ to point $B$ but not from $A$ to any other point, then $B$ is a terminal point of $M$.

CoRollary 2. If $M$ is a compact hereditarily unicoherent continuum irreducible from point $A$ to point $B$ and the set $H$ of all points $x$ such that $M$ is irreducible from $A$ to $x$ is a continuum and $P$ is a terminal point of $H$, then $P$ is a terminal point of $M$.

TheOREm 3.4. A nondegenerate continuum every subcontinuum of which is unicoherent and decomposable has two terminal points.

Proof. Let $M$ denote such a continuum. A maximal irreducible subcontinuum of a subcontinuum $Z$ of $M$ will be called an $I$-subcontinuum of $Z$. Let $N$ denote an $I$-subcontinuum of $M$, and let $H$ and $K$ denote the $E$-subcontinua of $N$. Let $\alpha$ denote a well-ordered sequence whose terms are the subcontinua of $H$. There exists a well-ordered sequence $\beta$ such that (1) the first term of $\beta$ is $H$ if $H$ is degenerate and otherwise the first term of $\alpha$ which is an $I$-subcontinuum of $H$; (2) every term of $\beta$ after the first is a proper subcontinuum of every one that precedes it; (3) if $X$ is a term of $\beta$ which is not a point, then it is an irreducible continuum, and, if $E_{X}$ is the first term of $\alpha$ which is an $E$-subcontinuum of $X$, the next term after $X$ in $\beta$ is $E_{X}$ if $E_{X}$ is degenerate and otherwise the first term of $\alpha$ which is an $I$-subcontinuum of $E_{X}$; and (4) if $\gamma$ is a segment $\left({ }^{18}\right)$ of $\beta$ which has no last term and $L$ is the common part of all the continua of $\gamma$, then the first term of $\beta$ following all those of $\gamma$ is $L$ if $L$ is degenerate and otherwise the first term of $\alpha$ which is an $I$-subcontinuum of $L$. The sequence $\beta$ has a last term and this last term is a point $A$. Suppose $A$ is not a terminal point of $M$. Then there exists an irreducible subcontinuum $Y$ of $M$ which contains $A$ and is not irreducible from $A$ to any point. By Theorem 3.3, $Y$ is a subset of $H$, and it follows from Theorems 3.2 and 3.3 that $Y$ is a subset of the first term of $\beta$. Since $A$ is the only point which is common to all the continua which are terms of $\beta$ and $\beta$ is well-ordered, there is a first term $U$ of $\beta$ which does not contain $Y$. Suppose that $U$ has an immediate predecessor $V$ in $\beta$. It follows from Theorem 3.3 that $U$ is nondegenerate, and therefore $U$ is an $I$-subcontinuum of an $E$-subcontinuum of $V$. Then, by Theorems 3.2 and 3.3, $U$ contains $Y$. From this contradiction it follows that $U$ has no predecessor in $\beta$. Let $L$ denote the common part of all the terms of $\beta$ which precede $U$. Since $L$ contains $Y$ it is nondegenerate. Then $U$ is an $I$-subcontinuum of an $E$-subcontinuum of $L$ and, by Theorems 3.2 and $3.3, U$ contains $Y$. From this contradiction it

(18) The well-ordered sequence $A$ is said to be a segment of the well-ordered sequence $B$ if either $A$ is $B$ or there exists a term $x$ of $B$ such that (1) $y$ is a term of $A$ if and only if it a term of $B$ which precedes $x$ in $B$, and (2) the term $y$ precedes the term $z$ in $A$ if and only if it precedes $z$ in $B$. 
follows that $A$ is a terminal point of $M$. Similarly $K$, which does not intersect $H$, contains a terminal point of $M$.

COROLlaRY 1. If $M$ is a nondegenerate continuum every subcontinuum of which is unicoherent and decomposable and $N$ is a maximal irreducible subcontinuum of $M$, then $N$ is irreducible between two points which are terminal points of $M$.

Corollary 2. A nondegenerate continuum $M$ every subcontinuum of which is unicoherent and decomposable is irreducible between two terminal points $A$ and $B$ such that of any two subcontinua of $M$ both containing $A$ (or $B$ ), one is a subset of the other.

TheOREm 3.5. A nondegenerate continuum every subcontinuum of which is unicoherent and decomposable is irreducible about the set of all its terminal points.

Proof. Let $M$ denote such a continuum. Suppose there exists a proper subcontinuum $N$ of $M$ which contains the set $H$ of all terminal points of $M$. Let $P$ be a point of $M-N$, and let $K$ denote a maximal irreducible subcontinuum of $M$ which contains $P$. By Corollary 1 of Theorem 3.4, $M$ has two terminal points $A$ and $B$ such that $K=A B$. Then $A$ and $B$ are in $N$ and hence $N$ contains $A B$. This involves a contradiction.

Theorem 3.6. If $M$ is a nondegenerate hereditarily decomposable continuum and $K$ is a subset of the set of all terminal points of $M$, then $M-K$ is connected.

Proof. Since $M$ is decomposable, it is clear that not every point of $M$ is a terminal point. Suppose that $M-K$ is the sum of two mutually separated sets $U$ and $V$. Let $A$ be a point of $U, B$ a point of $V$, and $H$ a subcontinuum of $M$ which is irreducible from $A$ to $B$. The continuum $H$ intersects $K$. Let $X$ and $Y$ be the $E$-subcontinua of $H$. The continuum $H$ is irreducible from each point of $H \cdot K$ to some point, and hence $H \cdot K$ is a subset of $X+Y$. Since $H-(X+Y)$ is connected, it is a subset of $U$ or of $V$. Since $A$ and $B$ belong to $X+Y$ and every point of a nondegenerate continuum is a limit point of each of its composants, $A$ and $B$ are limit points of $H-(X+Y)$, which is therefore not a subset of $U$ or of $V$. From this contradiction the theorem follows.

THEOREM 3.7. In order that a hereditarily decomposable continuum be an acyclic continuous curve it is necessary and sufficient that every point of it be either a cut point or a terminal point.

Proof. That the condition is necessary follows from well known properties of an acyclic continuous curve. 
Let $M$ denote a hereditarily decomposable continuum such that every point of $M$ is either a terminal point or a cut point. Let $N$ be any nondegenerate subcontinuum of $M$, and let $A$ and $B$ be two points of $N$. There is a subcontinuum $L$ of $N$ which is irreducible from $A$ to $B$. Let $H$ and $K$ denote the $E$-subcontinua of $L$. By Lemma A, $L-(H+K)$ is strongly connected. Then $L-(H+K)$ is an uncountable set, and no point of it is a terminal point of $M$. Hence $N$ contains uncountably many cut points of $M$ and, by Theorem 43 of Chapter II of Foundations, $M$ is an acyclic continuous curve.

Texas State College for Women,

Denton, Tex. 